\title{
Telehealth methods to deliver multifactorial dietary interventions in adults with chronic disease: a systematic review protocol
}

\author{
Jaimon T. Kelly ${ }^{1 *} \mathbb{D}$, Dianne P. Reidlinger ${ }^{1}$, Tammy C. Hoffmann² and Katrina L. Campbell ${ }^{1,3}$
}

\begin{abstract}
Background: The long-term management of chronic diseases requires adoption of complex dietary recommendations, which can be facilitated by regular coaching to support sustained behaviour change. Telehealth interventions can overcome patient-centred barriers to accessing face-to-face programs and provide feasible delivery methods, ubiquitous and accessible regardless of geographic location. The protocol for this systematic review explains the methods that will be utilised to answer the review question of whether telehealth interventions are effective at promoting change in dietary intake and improving diet quality in people with chronic disease.

Methods/design: A structured search of Medline, EMBASE, CINAHL, and PsychINFO, from their inception, will be conducted. We will consider randomised controlled trials which evaluate complex dietary interventions in adults with chronic disease. Studies must provide diet education in an intervention longer than 4 weeks in duration, and at least half of the intervention contact must be delivered via telehealth. Comparisons will be made against usual care or a nontelehealth intervention. The primary outcome of interest is dietary change with secondary outcomes relating to clinical markers pre-specified in the methodology. The process for selecting studies, extracting data, and resolving conflicts will follow a set protocol. Two authors will independently appraise the studies and extract the data, using specified methods. Meta-analyses will be conducted where appropriate, with parameters for determining statistical heterogeneity prespecified. The GRADE tool will be used for determining the quality of evidence for analysed outcomes.
\end{abstract}

Discussion: To date, there has been a considerable variability in the strategies used to deliver dietary education, and the overall effectiveness of telehealth dietary interventions for facilitating dietary change has not been reviewed systematically in adults with chronic disease. A systematic synthesis of telehealth strategies will inform the development of evidencebased telehealth programs that can be tailored to deliver dietary interventions specific to chronic disease conditions.

Systematic review registration: PROSPERO CRD42015026398

Keywords: Telehealth, Dietary, Diet quality, Adults, Chronic disease, Behaviour change, Lifestyle change, Technology

\section{Background}

Non-pharmacological treatment methods are commonly used for people with chronic diseases [1], and many of these require lifetime adherence to dietary recommendations. Telehealth technologies can provide education and self-management support to facilitate and sustain lifestyle changes. Such interventions (which include telephone

\footnotetext{
*Correspondence: jkelly@bond.edu.au

${ }^{1}$ Faculty of Health Science and Medicine, Bond University, Gold Coast, Australia

Full list of author information is available at the end of the article
}

coaching, the Internet, mobile phone applications) could have advantages over traditional face-to-face models of care [2], and utilisation of them may assist with achieving dietary behaviour change [2-4].

\section{Description of the condition}

Chronic disease is the leading cause of ill health, accounting for $68 \%$ of all deaths worldwide [5], in some countries contributing to over $90 \%$ of all deaths [6]. Chronic diseases are those with multifactorial aetiologies, and once diagnosed, are with the individual for life without a specific cure. Many chronic diseases are

\section{Biomed Central}


diet-related, specifically obesity, heart disease, diabetes, hypertension, stroke, and kidney disease as previously defined in a systematic review [7]. These pose a significant challenge to the health system, in terms of costs and cause of death and disability, which tends to be related to cardiovascular disease (CVD) as either the primary or co-morbid condition [8]. Self-management and the adoption of a healthy lifestyle, such as through diet, physical activity, and other health-related behaviours (e.g. smoking cessation), are considered essential for the management of these diet-related chronic diseases $[9,10]$.

Telephone-delivered interventions for smoking cessation [11] show improvements in quit rates, and physical activity [2] interventions delivered at least $50 \%$ by telephone show increases in all measures of physical activity, which is also sustained following the conclusion of interventions. However, the findings from studies aimed at increasing adherence to specific dietary recommendations in chronic disease lack consistent findings in controlled studies [12].

\section{Description of the intervention}

According to the World Health Organisation [13], the definition of telehealth is encompassed by the definition of telemedicine, which refers to the delivery of healthcare services at a distance, using information and communication technologies to exchange health information. A distinguishing characteristic of telemedicine is that it is restricted to healthcare delivery by physicians only, whereas telehealth services are provided by any health professional and can include either synchronous (i.e. same time, different location) and asynchronous (i.e. different time, different location) patient education, counselling, and remote monitoring [13]. A telehealth lifestyle intervention may involve the provision of lifestyle education or advice to an individual or group of individuals remotely via the telephone [14], computer, and the Internet [15-17], videos [18], email [19], and/or mobile phone applications including text, photo messages (short message service (SMS), or multimedia message service (MMS)) [20, 21].

\section{How the intervention might work}

For lifestyle interventions to achieve long-term behaviour change, an intensive approach which involves frequent engagement and ongoing monitoring is recommended [1, 9, 12]. This is particularly true for changes that require the long-term maintenance of dietary strategies, which require a high degree of self-management and are notorious for poor compliance and high participant burden [22]. To improve compliance, regular contact with treating clinicians and an emphasis on self-monitoring have been suggested as central to the success of a complex dietary intervention [23, 24]. Interventions which use telehealth strategies offer expedient and feasible ways to provide the recommended support to individuals to facilitate behaviour change. For example, individuals who have limited time to attend face-to-face education could access an education program from the comfort of their own home at a time of their choosing [25]. An advantage of telehealth interventions is that educational content can be provided live (synchronous) between patient and health professional, or through text messages, emails, and Internet outlets (asynchronous and mobile health), thus overcoming some of the common barriers to face-to-face care.

\section{Why it is important to do this review}

Technology to deliver health-related interventions have been used for over 25 years with mixed results, ranging from no effect at all to significant improvements in health outcomes [26]. Despite a range of telehealth methods for the management of chronic disease [2, 8, 27-31], as well as CVD risk behaviours [8], the effectiveness of telehealth interventions to facilitate dietary change has not been systematically synthesised. A recent systematic review demonstrated that telephone coaching is feasible for establishing effective behavioural change for physical activity and/or dietary interventions [2]. However, only two dietary studies met the inclusion criteria for this review, and studies were not specific to chronic disease. A recent systematic review demonstrated that telephone coaching is feasible for establishing effective behavioural change for physical activity and/or dietary interventions [2]. However, only two dietary studies met the inclusion criteria for this review, and studies were not specific to chronic disease. Another (Cochrane) review investigated the effectiveness of different interventions, in promoting adherence to dietary recommendations [12]. However in this review, although some included studies may have used telehealth as a component of the intervention, they did not evaluate the effectiveness of telehealth interventions specifically and did not compare to usual care (defined in its broadest sense, and which could include non-telehealth-delivered dietary advice from a health professional or no dietary education at all).

Despite a number of previous systematic reviews covering different combinations of telehealth and/or population groups (healthy and chronic disease), these reviews have not specifically evaluated interventions that attempt to change dietary patterns (i.e. multiple food groups or nutrients) which represent the dietary management of chronic disease [32].

Recent technology-based trials educating to the dietary guidelines (via the telephone) [14] and the DASH diet (via the Internet) [33] showed significant improvements in measures of dietary intake (such as fruit and vegetable consumption) for strategies using technology compared 
to those using more traditional strategies. Such evidence is promising for future healthcare as it may inform the development of evidence-based telehealth programs, which can be tailored to specific chronic disease conditions and may provide policy makers with more efficient options for funding programs for chronic disease management.

Although promising, to establish the effectiveness of telehealth interventions and inform future programs, telehealth interventions need to be systematically evaluated against these traditional educational strategies and to standard care alone to prompt changes in healthcare policy that have been long suggested to deal with lacking compliance to lifestyle recommendations and other barriers in current chronic disease healthcare [10].

However, there is no existing or registered systematic review that has sought to assess the overall effectiveness of telehealth dietary interventions for facilitating complex dietary change in adults with chronic disease to date.

\section{Objective}

The objective of this review is to assess the effectiveness of telehealth as a strategy to deliver complex dietary interventions in adults with chronic disease.

\section{Methods/design \\ Eligibility criteria \\ Study designs}

Eligible designs will be randomised controlled trials (RCTs), cluster RCTs, and quasi-RCTs (RCTs using pseudorandomisation). Trials which use crossover designs can introduce potential carry-over effects given the nature of dietary interventions to establish behaviour change; therefore, we will only include data from the first period of each intervention arm [34].

\section{Participants}

Adult participants ( $>18$ years of age) with an established diet-related chronic disease which we define as obesity (BMI $\geq 30 \mathrm{~kg} / \mathrm{m}^{2}$ ), diabetes mellitus, established heart disease, hypertension, stroke, and kidney disease. These diet-related chronic diseases have been previously defined in a systematic review of dietary interventions [12]. We will review studies that report on a mixed sample, however, will only include participants with a chronic disease as defined above, and provided their results are reported separately to participants that do not meet our inclusion criteria.

\section{Interventions}

Eligible interventions will be those that provide a multifactorial dietary intervention using at least one telehealth strategy with a duration of at least 4 weeks. We define a multifactorial dietary intervention as targeting more than a singular nutrient and/or food group. Multifactorial dietary interventions include those aimed at overall dietary patterns, such as dietary guidelines $[35,36]$, the Mediterranean diet [37], and/or the DASH diet or those educating on two or more dietary components (nutrients and/or food groups) simultaneously. Studies that target two or more diet changes within the same nutrient (e.g. manipulation of categories of fatty acids) will be excluded as the dietary components only relate to one nutrient, and thus are not multifactorial.

Interventions that use either a single or multifactorial telehealth strategy will be eligible. Interventions that use both telehealth and non-telehealth strategies (e.g. faceto-face, group counselling) to provide dietary education will be eligible as long as at least $50 \%$ of the total contact hours and/or the total number of interaction contacts with participants are delivered via telehealth methods. An example of an interaction is a text message, a phone call, log-on to a webpage, or a contact session with an intervention provider. Eligible interventions will be delivered by a qualified healthcare professional (such as a nurse, dietitian, or physician).

\section{Comparators}

The comparison group may have received usual care (as defined by trial authors); dietary education in a face-toface or group-based environment with no telehealth component, or via a method in which less than $50 \%$ of the intervention is delivered via telehealth; or a nondietary focussed intervention.

\section{Outcomes}

We will only include studies that report at least two measures of dietary intake: at baseline and at least 4 weeks later at follow-up.

Primary outcomes:

- Dietary intake: any objective measure of dietary intake (such as diet quality score, servings of fruits and vegetables, and nutrient intake)

Although surrogate outcomes such as dietary intake cannot reliably predict clinical outcomes (e.g. mortality), dietary intake is the first line management strategy in chronic diseases [10]. It is clinically relevant to chronic disease as it may improve clinical outcomes and is a practical policy tool to inform the development of evidence-based telehealth programs, particularly for the chronic diseases listed above. Dietary intake is measured in a variety of units, and we have chosen not to restrict our primary outcome by unit of measure. Furthermore, this review may identify which outcome measures of 
dietary intake are stronger as surrogate markers of our secondary clinical outcomes.

Secondary outcomes:

- All-cause mortality;

- Cardiovascular mortality;

- Hospitalisations (total and those related to chronic disease);

- Any clinical marker of chronic disease progression, such as blood pressure, estimated glomerular filtration rate (eGFR), HbA1c, weight, waist circumference, and blood lipid profiles.

\section{Setting}

Studies will be eligible if the intervention is conducted in ambulatory or community settings (e.g. patients can be recruited during a hospital admission, but the telehealth intervention is delivered post-discharge). Studies that are solely conducted in hospitals or controlled conditions (e.g. where food and/or beverages are provided in full or partial) will not be eligible.

\section{Language}

No language restriction will be in our search strategy. We will attempt to translate potentially eligible nonEnglish articles via Google Translate or by a native speaker of the language of the article. In the event that an article is eligible but unable to be satisfactorily translated, we will present the title and author details in a supplementary appendix.

\section{Search methods}

\section{Electronic searches}

A multi-step search approach will be undertaken to retrieve relevant studies. The following databases will be searched using a variety of subject headings, free text terms, and synonyms relevant to the review in consultation with an experienced search trials co-ordinator (see Additional file 1):

- MEDLINE (via OVID);

- CINAHL (via EBSCO);

- PsychINFO (via OVID); and

- EMBASE.

- Searches of the International Clinical Trials Register (ICTRP) Search Portal and ClinicalTrials.gov will be conducted to identify trials that are ongoing.

We will perform forward and backward citation searching of eligible studies. We will attempt to locate unpublished trials by contacting investigators known to be involved in previous studies that have not yet been published and by contacting published authors in the field of telehealth research and asking if they are aware of ongoing and unpublished trials.

Finally, we will perform a search for relevant theses and dissertations (via ProQuest) and conference abstracts (such as the annual meetings for the American Telemedicine Association, the International Conference on Health Informatics, and the Australasian Telehealth Society).

\section{Selection of studies}

All search results will be merged into reference management software EndNote, and duplicate records of the same report will be removed using the Centre for Research and Evidence Based Practice Systematic Review Assistant 'deduplication tool' [38]. Two review authors ( $\mathrm{KK}$ and $\mathrm{KC}$ ) will independently assess the eligibility of studies by screening titles and abstracts for potential inclusion according to predefined selection criteria. Studies judged to be potentially relevant will be retrieved in full text for further analysis. Any disagreements in judgement will be resolved by discussion to reach a consensus or if this is not possible, with a third review author (DR) until a consensus is reached. If further information about the study is required in order to make a decision about its eligibility, an attempt will be made to contact the study corresponding author(s). If a response is not received after three reminders are sent and/or after attempting to contact another author of the paper with no response, then the study will be excluded.

\section{Data collection and analysis \\ Data extraction and management}

Two independent authors will extract the data independently (JK and $\mathrm{KC}$ ). Data will be extracted from all published reports of included studies using a data extraction form which will be piloted following adaptation from the Cochrane Effective Practice and Organisation of Care Group tool [39]. For all included studies, we will extract relevant data including all details about the intervention (following the components outlined in the Template for Intervention Description and Replication (TIDieR) checklist) [40], the participants (chronic disease state, age, and gender), attrition, and all our prespecified primary and secondary outcome data that are reported at baseline and follow-up. All extracted data will be transferred into Revman software (JK) and will be checked for accuracy (KC) prior to meta-analysis.

\section{Assessing the risk of bias}

Risk of bias will be assessed by two review authors (JK \& $\mathrm{KC}$ ) using the Cochrane risk of bias tool addressing the following elements that potentially affect risk of bias:

- Random sequence generation;

- Allocation concealment; 
- Blinding-clients, providers, and outcome assessors;

- Incomplete outcome data;

- Selective reporting;

- Other bias.

Any disagreements in judgement will be resolved by discussion to reach a consensus or with a third review author until consensus is reached. We will tabulate and narratively describe the risk of bias in the included studies. For each study, we will categorise the risk of bias elements as low, unclear, or high risk. The effect that studies with a high risk of bias may have on the body of evidence will be explored in sensitivity analyses described below. We will consider the risk of bias for each outcome when grading the quality of the evidence.

\section{Data analysis}

The overall treatment effect for primary and secondary outcomes will be calculated from each trial included. The treatment effect will be calculated, where possible, as the difference between the intervention and comparison's change from baseline to the end of follow-up for each of the measured outcomes. Variance will be calculated for each treatment effect, either derived from the standard deviation or standard error from the difference between baseline and follow-up or from confidence intervals when these are not available [34].

\section{Measures of treatment effect}

Where the studies included have reported interventions and outcomes which are sufficiently homogeneous, and if there is sufficient information retrieved from the studies, quantitative data will be pooled into Revman (Version 5.3) for meta-analysis using the DerSimonian and Laird random-effects model [41]. Fixed-effect model will also be used to ensure robustness and susceptibility to outliers. Effect sizes (for continuous data; e.g. diet intake, biomarkers, blood pressure, and weight) will be calculated as mean differences (MD) or as the standardised mean difference (SMD) if different scales have been used, and their $95 \%$ confidence intervals will be calculated to measure the treatment effect. The ratio of means (RoMs) is an alternate method for data pooling $[42,43]$ and will be alternatively used if the SMD cannot be calculated from the outcome measures extracted from the included studies. We will convert other forms of data into MD, SMD, or RoMs and calculate confidence intervals as required. Dichotomous outcome data (e.g. death, hospitalisations, and progression to renal replacement therapy) will be expressed as risk ratios (RR) with $95 \%$ confidence intervals. We will convert other relevant binary data into an RR value. In the event of missing data, we will attempt to impute missing standard deviations or standard errors using data from other similar studies in the review utilising similar methods and sample sizes, as recommended [44].

\section{Assessment of heterogeneity}

We will assess the inconsistencies between studies using the $I^{2}$ statistic and describe the percentage of variability in effect. Heterogeneity will be considered substantial if the $I^{2}$ statistic is $>50 \%$. We will use Egger's plot to assess and report on potential publication bias. We will consider a sensitivity analysis to explore statistical heterogeneity. The sensitivity analysis will be considered if the results of an individual study appear to be heterogeneous with the results of other included studies; repeat the analysis excluding unpublished studies; repeat the analysis excluding high risk of bias studies (method of randomisation, allocation concealment, blinding of outcome assessor, incomplete outcome data, selective reporting, other bias); and repeat the analysis excluding any long duration studies or large studies in order to establish how much they influence effect sizes.

\section{Subgroup analyses}

Depending on the included studies, we will conduct subgroup analysis to explore expected sources of clinical heterogeneity. Subgroup analyses will be considered on studies conducted in those with different chronic health conditions (e.g. diabetes, established heart disease, chronic kidney disease, and hypertension); studies using different telehealth strategies (e.g. Internet or mobile phone); studies with multiple telehealth modes of delivery (e.g. Internet and telephone) versus single mode; studies greater than 6 months versus less than 6 months duration; studies where dietary education is provided in the comparison group; studies targeting specified food groups or multiple nutrients (e.g. modifying sodium, fat, fruit and/or vegetables interventions) versus dietary patterns; and studies where dietary intervention is either the sole focus of an intervention versus as part of a complex multicomponent intervention (i.e. diet and exercise).

\section{Presenting and reporting of results}

This protocol follows the Preferred Reporting Items for Systematic Review and Meta-Analysis Protocols (PRISMAP) 2015 Statement [45] (see Additional file 2). We will present the results of this review according to the Preferred Reporting Items for Systematic Reviews and Meta-Analyses (PRISMA) guidelines, using a flow diagram to report the identification and selection of studies, and assign a grading to the evidence using the GRADE tool. The relevant outcomes and characteristics of each study will be reported in summary tables. Where statistical pooling is not possible, the findings will be alternatively presented in a narrative form including tables and figures to aid in data presentation 
where appropriate. We will follow the Cochrane handbook guidelines for narrative synthesis, whereby grouping similar studies under headings (e.g. similar telehealth methods, dietary education, comparator studied (usual care and other dietary education delivery methods), chronic disease, dietary outcome measures) and report the direction, size and consistency of effect, and the overall quality of the body of evidence. For trials with more than one time point measurement for outcomes, we will only report results extracted from the furthest time points of the intervention.

\section{Interpretation of findings}

The results of the review will be discussed in the context of the quality of the evidence (GRADE), the limitations of the review, and the strengths of findings as well as their implications for current practice, future directions, and overall public health. To interpret the overall effectiveness of telehealth interventions and allow policy makers to effectively determine their efficacy at facilitating multifactorial dietary changes, we will also discuss the results in the context of the comparator studied (usual care and other dietary education delivery methods) as necessary given the retrieved body of evidence.

\section{Discussion}

This protocol for a systematic review of available evidence will establish whether telehealth is an effective strategy to deliver multifactorial dietary interventions in adults with chronic disease, which has not been previously evaluated or reviewed systematically. If telehealth is found to be effective in establishing multifactorial dietary change, this may inform a change in current clinical and public health practice by restructuring funding and resources for future chronic disease dietary management in healthcare. Furthermore, the primary results of this review and any long-term adverse consequences found by the review may be used to inform the development of evidence-based telehealth programs, best practice guidelines, and recommendations for future telehealth intervention delivery, which can be tailored to specific chronic disease conditions. This review will also identify any apparent gaps in the body of literature and highlight priorities for future research in the area.

\section{Additional files}

Additional file 1: Search strategy. Additional file 1 presents the MEDLINE search strategy which will be used to identify potential studies. (PDF $153 \mathrm{~kb}$ )

Additional file 2: PRISMA-P checklist. Additional file 2 presents the PRISMA-P checklist. (PDF $152 \mathrm{~kb}$ )

\section{Abbreviations}

BMl: body mass index; CVD: cardiovascular disease; DASH: dietary approaches to stop hypertension; eGFR: estimated glomerular filtration rate; GRADE: grading of recommendations assessment, development and evaluation; HbA1c: glycated haemoglobin; ICTRP: International Clinical Trials Register; MD: mean difference; MMS: multimedia message service; PRISMA: Preferred Reporting Items for Systematic Review and Meta-Analysis; PRISMA-P: Preferred Reporting Items for Systematic Review and Meta-Analysis Protocols; Quasi-RCT: quasi-experimental controlled trial; RCT: randomised controlled trial; RoMs: ratio of means; RR: relative risk; SD: standard deviation; SMD: standardised mean difference; SMS: short message service; TIDieR: template for intervention description and replication.

\section{Competing interests}

The authors declare that they have no competing interests.

\section{Authors' contributions}

JK and KC were responsible for conducting the review and subsequent analysis. JK assisted in the conceptualisation of the review and drafted the study protocol. KC conceived the review and revised manuscript drafts. DR assisted in the conceptualisation of the review and reviewed all drafts of the manuscript. TH participated in the design of the review, provided methodological expertise, and reviewed drafts of the manuscript. All authors read and approved the final manuscript.

\section{Acknowledgements}

This work has received no specific funding. JK is supported by an Australian Post Graduate Award scholarship through Bond University. The authors wish to thank Mr David Honeyman and Mr Justin Clark for their assistance in the development of the search strategy. Thank you to the peer reviewers whose comments and feedback into this manuscript has strengthened the review protocol.

\section{Author details}

${ }^{1}$ Faculty of Health Science and Medicine, Bond University, Gold Coast, Australia. ${ }^{2}$ Centre for Research in Evidence Based Practice, Faculty of Health Sciences and Medicine, Bond University, Gold Coast, Australia. ${ }^{3}$ Nutrition and Dietetics Department, Princess Alexandra Hospital, Brisbane, Australia.

Received: 12 October 2015 Accepted: 14 December 2015

Published online: 22 December 2015

\section{References}

1. Lichtenstein AH, Appel LJ, Brands M, Carnethon M, Daniels S, Franch HA et al. Diet and lifestyle recommendations revision 2006: a scientific statement from the American Heart Association nutrition committee. Circulation. 2006;114(1):82-96.

2. Goode AD, Reeves MM, Eakin EG. Telephone-delivered interventions for physical activity and dietary behavior change: an updated systematic review. Am J Prev Med. 2012;42(1):81.

3. Direito A, Dale LP, Shields E, Dobson R, Whittaker R, Maddison R. Do physical activity and dietary smartphone applications incorporate evidencebased behaviour change techniques? BMC Pub Health. 2014;14(1):646.

4. Norman GJ, Zabinski MF, Adams MA, Rosenberg DE, Yaroch AL, Atienza AA. A review of eHealth interventions for physical activity and dietary behavior change. Am J Prev Med. 2007:33(4):336-45. e16.

5. Mendis S. Global status report on noncommunicable diseases 2014. In. World Health Organization, 2014. http://www.who.int/nmh/publications/ ncd-status-report-2014/en/. Accessed 4 July 2015

6. Australian Institute of Health and Welfare. Australia's health 2014. In: Australia's health series no. 14. Canberra: AlHW; 2014. Cat. no. AUS 178.

7. WHO J, Consultation FE. Diet, nutrition and the prevention of chronic diseases. WHO Tech Rep Ser. 2003;916:1-60.

8. Burke LE, Ma JZ, Azar KMJ, Bennett GG, Peterson ED, Zheng Y, et al. Current science on consumer use of mobile health for cardiovascular disease prevention a scientific statement from the American Heart Association. Circulation. 2015. doi:10.1161/CIR.0000000000000232.

9. Celler BG, Lovell NH, Basilakis J. Using information technology to improve the management of chronic disease. Med J Aust. 2003;179(5):242-6.

10. Artinian NT, Fletcher GF, Mozaffarian D, Kris-Etherton P, Van Horn L, Lichtenstein $\mathrm{AH}$, et al. Interventions to promote physical activity and dietary lifestyle changes for cardiovascular risk factor reduction in adults a scientific statement from the American Heart Association. Circulation. 2010;122(4):406-41. 
11. Stead LF, Perera R, Lancaster T. Telephone counselling for smoking cessation. Cochrane Database Syst Rev. 2006;3:CD002850.

12. Desroches S, Lapointe A, Ratte S, Gravel K, Legare F, Turcotte S. Interventions to enhance adherence to dietary advice for preventing and managing chronic diseases in adults. Cochrane Database Syst Rev. 2013;2:Cd008722.

13. World Health Organization. Telemedicine opportunities and developments in member states: report on the second global survey on eHealth. In: Global observatory for eHealth series_-volume 2. Switzerland: World Health Organisation; 2010

14. Eakin E, Reeves M, Lawler S, Graves N, Oldenburg B, Del Mar C, et al. Telephone counseling for physical activity and diet in primary care patients. Am J Prev Med. 2009;36(2):142-9.

15. Touger-Decker R, Denmark R, Bruno M, O'Sullivan-Maillet J, Lasser N. Workplace weight loss program; comparing live and internet methods. J Occup Environ Med/Am Coll Occup Environ Med. 2010;52(11):1112-8.

16. McConnon A, Kirk SF, Cockroft JE, Harvey EL, Greenwood DC, Thomas JD, et al. The Internet for weight control in an obese sample: results of a randomised controlled trial. BMC Health Serv Res. 2007;7(1):206.

17. Tate DF, Wing RR, Winett RA. Using Internet technology to deliver a behavioral weight loss program. JAMA. 2001;285(9):1172-7.

18. Cook RF, Billings DW, Hersch RK, Back AS, Hendrickson A. A field test of a web-based workplace health promotion program to improve dietary practices, reduce stress, and increase physical activity: randomized controlled trial. J Med Internet Res. 2007;9(2):e17.

19. Dorough AE, Winett RA, Anderson ES, Davy BM, Martin EC, Hedrick V. DASH to wellness: emphasizing self-regulation through E-health in adults with prehypertension. Health Psychol. 2014;33(3):249-54.

20. Nundy S, Razi RR, Dick JJ, Smith B, Mayo A, O'Connor A, et al. A text messaging intervention to improve heart failure self-management after hospital discharge in a largely African-American population: before-after study. J Med Internet Res. 2013;15(3):e53.

21. Morikawa N, Yamasue K, Tochikubo O, Mizushima S. Effect of salt reduction intervention program using an electronic salt sensor and cellular phone on blood pressure among hypertensive workers. Clinical and experimental hypertension (New York. NY. 1993;2011.33(4):216-22.

22. Jerant AF, von Friederichs-Fitzwater MM, Moore M. Patients' perceived barriers to active self-management of chronic conditions. Patient Educ Couns. 2005:57(3):300-7.

23. Greaves CJ, Sheppard KE, Abraham C, Hardeman W, Roden M, Evans PH, et al. Systematic review of reviews of intervention components associated with increased effectiveness in dietary and physical activity interventions. BMC Pub Health. 2011;11(1):119.

24. Renders CM, Valk GD, Griffin S, Wagner EH, Eijk JT, Assendelft WJ. Interventions to improve the management of diabetes mellitus in primary care, outpatient and community settings. Cochrane Database Syst Rev. 2001;1:CD001481.

25. Southard BH, Southard DR, Nuckolls J. Clinical trial of an Internet-based case management system for secondary prevention of heart disease. J Cardiopulm Rehabil. 2003;23(5):341-8.

26. Wootton R. Twenty years of telemedicine in chronic disease management-an evidence synthesis. J Telemed Telecare. 2012;18(4):211-20.

27. Beratarrechea A, Lee AG, Willner JM, Jahangir E, Ciapponi A, Rubinstein A. The impact of mobile health interventions on chronic disease outcomes in developing countries: a systematic review. Telemed J E-health. 2014;20(1):75-82.

28. Cassimatis M, Kavanagh DJ. Effects of type 2 diabetes behavioural telehealth interventions on glycaemic control and adherence: a systematic review. J Telemed Tel. 2012;18(8):447-50.

29. de Jongh T, Gurol-Urganci I, Vodopivec-Jamsek V, Car J, Atun R. Mobile phone messaging for facilitating self-management of long-term illnesses. Cochrane Database Syst Rev. 2012;12:CD007459.

30. Murray E, Burns JSST, See TS, Lai R, Nazareth I. Interactive health communication applications for people with chronic disease. Cochrane Database Syst Rev. 2005:4:CD004274.

31. Xiang R, Li L, Liu SX. Meta-analysis and meta-regression of telehealth programmes for patients with chronic heart failure. J Telemed Telecare. 2013;19(5):249-59.

32. Hu FB. Dietary pattern analysis: a new direction in nutritional epidemiology. Curr Opin Lipidol. 2002;13(1):3-9.

33. Green BB, Anderson ML, Cook AJ, Catz S, Fishman PA, McClure JB, et al. eCare for heart wellness: a feasibility trial to decrease blood pressure and cardiovascular risk. Am J Prev Med. 2014:46(4):368-77.
34. Higgins JPT, Green S. Cochrane handbook for systematic reviews of interventions: Wiley Online Library. 2008.

35. Cespedes EM, Hu FB. Dietary patterns: from nutritional epidemiologic analysis to national guidelines. Am J Clin Nutr. 2015;101(5):899-900.

36. USDA. Scientific report of the 2015 Dietary Guidelines Advisory Committee. 2015.

37. Panagiotakos DB, Pitsavos C, Arvaniti F, Stefanadis C. Adherence to the Mediterranean food pattern predicts the prevalence of hypertension, hypercholesterolemia, diabetes and obesity, among healthy adults; the accuracy of the MedDietScore. Prev Med. 2007:44(4):335-40.

38. Rathbone J, Carter M, Hoffmann T, Glasziou P. Better duplicate detection for systematic reviewers: evaluation of Systematic Review AssistantDeduplication Module. Syst Rev. 2015;4:6.

39. Effective Practice and Organisation of Care (EPOC). Data collection form EPOC resources for review authors. Oslo: Norwegian Knowledge Centre for the Health Services; 2013.

40. Hoffmann TC, Glasziou PP, Boutron I, Milne R, Perera R, Moher D, et al. Better reporting of interventions: template for intervention description and replication (TIDieR) checklist and guide. BMJ. 2014;348:g1687.

41. DerSimonian R, Laird N. Meta-analysis in clinical trials. Control Clin Trials. 1986;7(3):177-88.

42. Friedrich JO, Adhikari NKJ, Beyene J. The ratio of means method as an alternative to mean differences for analyzing continuous outcome variables in meta-analysis: a simulation study. BMC Med Res Methodol. 2008;8(1):32.

43. Friedrich JO, Adhikari NKJ, Beyene J. Ratio of means for analyzing continuous outcomes in meta-analysis performed as well as mean difference methods. J Clin Epidemiol. 2011;64(5):556-64.

44. Wiebe N, Vandermeer B, Platt RW, Klassen TP, Moher D, Barrowman NJ. A systematic review identifies a lack of standardization in methods for handling missing variance data. J Clin Epidemiol. 2006;59(4):342-53.

45. Moher D, Shamseer L, Clarke M, Ghersi D, Liberati A, Petticrew M, et al. Preferred reporting items for systematic review and meta-analysis protocols (PRISMA-P) 2015 statement. Syst Rev. 2015;4(1):1.

\section{Submit your next manuscript to BioMed Central and we will help you at every step:}

- We accept pre-submission inquiries

- Our selector tool helps you to find the most relevant journal

- We provide round the clock customer support

- Convenient online submission

- Thorough peer review

- Inclusion in PubMed and all major indexing services

- Maximum visibility for your research

Submit your manuscript at www.biomedcentral.com/submit 